VOL. 73 (2006) [263-272]

\title{
NAMIOKA SPACES AND TOPOLOGICAL GAMES
}

\author{
V.V. MYKHAYLYUK
}

We introduce a class of $\beta-v$-unfavourable spaces, which contains some known classes of $\beta$-unfavourable spaces for topological games of Choquet type. It is proved that every $\beta-v$-unfavourable space $X$ is a Namioka space, that is for any compact space $Y$ and any separately continuous function $f: X \times Y \rightarrow \mathbb{R}$ there exists a dense in $X$ $G_{\delta}$-set $A \subseteq X$ such that $f$ is jointly continuous at each point of $A \times Y$.

\section{INTRODUCTION}

Investigations of the joint continuity point set of separately continuous functions were started by Baire in [2] and were continued in papers of many mathematicians. A Namioka's result [7] on a massivity of the joint continuity point set of separately continuous function on the product of two topological spaces, one of which satisfies compactness type conditions, gave a new impulse to a further investigation of this topic.

A topological space $X$ is called a strongly countably complete space if there exists a sequence $\left(\mathcal{U}_{n}\right)_{n=1}^{\infty}$ of open coverings of $X$ such that $\bigcap_{n=1}^{\infty} F_{n} \neq \emptyset$ for every centred sequence $\left(F_{n}\right)_{n=1}^{\infty}$ of closed in $X$ sets $F_{n}$ with $F_{n} \subseteq U_{n}$ for every $n \in \mathbb{N}$ and some $U_{n} \in \mathcal{U}_{n}$.

THEOREM 1.1. (Namioka.) Let $X$ be a strongly countably complete space, $Y$ be a compact space and $f: X \times Y \rightarrow \mathbb{R}$ be a separately continuous function. Then there exists a dense in $X G_{\delta}$-set $A \subseteq X$ such that $f$ is jointly continuous at each point of $A \times Y$.

The following notions were introduced in [9].

A mapping $f: X \times Y \rightarrow \mathbb{R}$ has the Namioka property if there exists a dense in $X$ $G_{\delta}$-set $A \subseteq X$ such that $A \times Y \subseteq C(f)$, where $C(f)$ means the joint continuity point set of $f$.

A Baire space $X$ is called a Namioka space if for any compact space $Y$, every separately continuous function $f: X \times Y \rightarrow \mathbb{R}$ has the Namioka property.

It was shown in [4] that a topological games technique can be useful in a study of Namioka spaces.

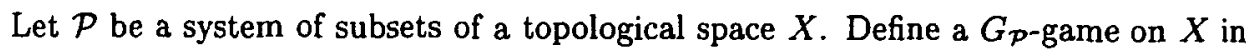
which two players $\alpha$ and $\beta$ participate. A nonempty open in $X$ set $U_{0}$ is the first move

Received 21st November, 2005

Copyright Clearance Centre, Inc. Serial-fee code: 0004-9727/06 \$A2.00+0.00. 
of $\beta$ and a nonempty open in $X$ set $V_{1} \subseteq U_{0}$ and set $P_{1} \in \mathcal{P}$ are the first move of $\alpha$. Further $\beta$ chooses a nonempty open in $X$ set $U_{1} \subseteq V_{1}$ and $\alpha$ chooses a nonempty open in $X$ set $V_{2} \subseteq U_{1}$ and a set $P_{2} \in \mathcal{P}$ and so on. The player $\alpha$ wins if

$$
\left(\bigcap_{n=1}^{\infty} V_{n}\right) \cap\left(\overline{\left(\bigcup_{n=1}^{\infty} P_{n}\right.}\right) \neq \emptyset
$$

Otherwise $\beta$ wins.

A topological space $X$ is called $\alpha$-favourable in the $G_{\mathcal{P}}$-game if $\alpha$ has a winning strategy in this game. A topological space $X$ is called $\beta$-unfavourable in the $G_{\mathcal{p}}$-game if $\beta$ has no winning strategy in this game. Clearly, any $\alpha$-favourable topological space $X$ is a $\beta$-unfavourable space.

In the case of $\mathcal{P}=\{X\}$ the game $G_{\mathcal{P}}$ is the classical Choquet game and $X$ is $\beta$ unfavourable in this game if and only if $X$ is a Baire space (see [9]). If $\mathcal{P}$ is the system of all finite (or one-point) subsets of $X$ then $G_{\mathcal{P}}$-game is called a $\sigma$-game.

Note that Christensen in [4] generalising the Namioka theorem considered an $s$-game which is a modification of the $\sigma$-game. So, for the $s$-game $\alpha$ and $\beta$ play in the same way as in the $\sigma$-game, and $\alpha$ wins if each subsequence $\left(x_{n_{k}}\right)_{k=1}^{\infty}$ of sequence $\left(x_{n}\right)_{n=1}^{\infty}$ has a cluster point in the set $\bigcap_{n=1}^{\infty} V_{n}$, where $P_{n}=\left\{x_{n}\right\}$. It was proved in [4] that any $\alpha-s$-favourable space is a Namioka space.

Saint-Raymond showed in [9] that for usage the topological games method in these investigations it is enough to require a weaker condition of $\beta$-unfavourability instead of the $\alpha$-favourability. He proved that any $\beta-\sigma$-unfavourable space is a Namioka space and generalised the Christensen result.

A further development of this technique leads to a consideration of another topological games which based on wider systems $\mathcal{P}$ of subsets of a topological space $X$.

Let $T$ be a topological space and $\mathcal{K}(T)$ be a collection of all compact subsets of $T$. Then $T$ is said to be $\mathcal{K}$-countably-determined if there exist a subset $S$ of the topological space $\mathbb{N}^{N}$ and a mapping $\varphi: S \rightarrow \mathcal{K}(T)$ such that for every open in $T$ set $U \subseteq T$ the set $\{s \in S: \varphi(s) \subseteq U\}$ is open in $S$ and $T=\bigcup_{s \in S} \varphi(s)$; and it is called $\mathcal{K}$-analytical if there exists such a mapping $\varphi$ for the set $S=\mathbb{N}^{N}$.

A set $A$ in a topological space $X$ is called bounded if for any continuous function $f: X \rightarrow \mathbb{R}$ the set $f(A)=\{f(a): a \in A\}$ is bounded.

The following theorem gives further generalisations of Saint-Raymond result.

THEOREM 1.2. Any $\beta$-unfavourable in $G_{\mathcal{P}}$-game topological space $X$ is a Namioka space if:

(i) $\mathcal{P}$ is the system of all compact subsets of $X$ (see Talagrand [11]);

(ii) $\mathcal{P}$ is the system of all $\mathcal{K}$-analytical subsets of $X$ (see Debs [5]);

(iii) $\mathcal{P}$ is the system of all bounded subsets of $X$ (see Maslyuchenko [6]); 
(iv) $\mathcal{P}$ is a system of all $\mathcal{K}$-countable-determined subsets of $X$ (see Rybakov [8]).

It is easy to see that (iv) $\Rightarrow$ (ii) $\Rightarrow$ (i) and (iii) $\Rightarrow$ (i).

In this paper, using a technique which is related to the dependence of functions on products upon some quantity of coordinates, we prove a result which generalises (iii) and (iv).

\section{DEPENDENCE OF FUNCTIONS UPON SOME QUANTITY OF COORDINATES AND}

\section{NAMIOKA PROPERTY}

Proposition 2.1. Let $X$ be a topological space, $A \subseteq X$ be a dense in $X$ set, $Y \subseteq \mathbb{R}^{T}$ be a topological space, $f: X \times Y \rightarrow \mathbb{R}$ be a continuous in the first variable function, $\varepsilon \geqslant 0$ and $S \subseteq T$ be such that $\left|f\left(a, y^{\prime}\right)-f\left(a, y^{\prime \prime}\right)\right| \leqslant \varepsilon$ for every $a \in A$ and every $y^{\prime}, y^{\prime \prime} \in Y$ with $\left.y^{\prime}\right|_{S}=\left.y^{\prime \prime}\right|_{s}$. Then $\left|f\left(x, y^{\prime}\right)-f\left(x, y^{\prime \prime}\right)\right| \leqslant \varepsilon$ for every $x \in X$ and every $y^{\prime}, y^{\prime \prime} \in Y$ with $\left.y^{\prime}\right|_{S}=\left.y^{\prime \prime}\right|_{S}$.

Proof: Suppose that $y^{\prime}, y^{\prime \prime} \in Y$ with $\left.y^{\prime}\right|_{s}=\left.y^{\prime \prime}\right|_{s}$. Put

$$
h^{\prime}: X \rightarrow \mathbb{R}, h^{\prime}(x)=f\left(x, y^{\prime}\right), \quad h^{\prime \prime}: X \rightarrow \mathbb{R}, h^{\prime \prime}(x)=f\left(x, y^{\prime \prime}\right) .
$$

Since $h^{\prime}$ and $h^{\prime \prime}$ are continuous, the set

$$
G=\left\{x \in X:\left|h^{\prime}(x)-h^{\prime \prime}(x)\right|>\varepsilon\right\}
$$

is open. But $G \cap A=\emptyset$ and $\bar{A}=X$. Thus, $G=\emptyset$ and $f\left(x, y^{\prime}\right)=f\left(x, y^{\prime \prime}\right)$ for each $x \in X$.

PRoposition 2.2. Let $Y \subseteq \mathbb{R}^{T}$ be a compact space, $(Z,|\cdot-\cdot| z)$ be a metric space, $f: Y \rightarrow Z$ be a continuous mapping, $\varepsilon \geqslant 0$ and $S \subseteq T$ be such that $\mid f\left(y^{\prime}\right)$ $-\left.f\left(y^{\prime \prime}\right)\right|_{z} \leqslant \varepsilon$ for every $y^{\prime}, y^{\prime \prime} \in Y$ with $\left.y^{\prime}\right|_{s}=\left.y^{\prime \prime}\right|_{s}$. Then for every $\varepsilon^{\prime}>\varepsilon$ there exist a finite set $S_{0} \subseteq S$ and a real $\delta>0$ such that $\left|f\left(y^{\prime}\right)-f\left(y^{\prime \prime}\right)\right|_{z} \leqslant \varepsilon^{\prime}$ for every $y^{\prime}, y^{\prime \prime} \in Y$ with $\left|y^{\prime}(s)-y^{\prime \prime}(s)\right|<\delta$ for each $s \in S_{0}$.

ProOF: Fix some $\varepsilon^{\prime}>\varepsilon$. Suppose that the proposition is false for this $\varepsilon^{\prime}$. Put $A=\{(R, n): R \subseteq S$ is finite and $n \in \mathbb{N}\}$. Consider on $A$ the following order: $\left(R^{\prime}, n^{\prime}\right)$ $\leqslant\left(R^{\prime \prime}, n^{\prime \prime}\right)$ if $R^{\prime} \subseteq R^{\prime \prime}$ and $n^{\prime} \leqslant n^{\prime \prime}$. By the assumption, for every $a=(R, n) \in A$ there exist $y_{a}^{\prime}, y_{a}^{\prime \prime} \in Y$ such that $\left|f\left(y_{a}^{\prime}\right)-f\left(y_{a}^{\prime \prime}\right)\right|_{z}>\varepsilon^{\prime}$ and $\left|y_{a}^{\prime}(s)-y_{a}^{\prime \prime}(s)\right|<1 / n$ for each $s \in R$. Since $Y^{2}$ is a compact, the net $\left(y_{a}^{\prime}, y_{a}^{\prime \prime}\right)_{a \in A}$ has a subnet $\left(z_{b}^{\prime}, z_{b}^{\prime \prime}\right)_{b \in B}$ which converges in $Y^{2}$ to some point $\left(y^{\prime}, y^{\prime \prime}\right)$. The continuity of $f$ implies $\left|f\left(y^{\prime}\right)-f\left(y^{\prime \prime}\right)\right|_{Z} \geqslant \varepsilon^{\prime}>\varepsilon$. For every $a_{0}=\left(\left\{s_{0}\right\}, n_{0}\right) \in A$ there exists $b_{0} \in B$ such that $a \geqslant a_{0}$ for every $b \geqslant b_{0}$ where $a \in A$ is such that $\left(z_{b}^{\prime}, z_{b}^{\prime \prime}\right)=\left(y_{a}^{\prime}, y_{a}^{\prime \prime}\right)$. Therefore $\left|y^{\prime}\left(s_{0}\right)-y^{\prime \prime}\left(s_{0}\right)\right| \leqslant 1 / n_{0}$ for every $s_{0} \in S$ and $n_{0} \in \mathbb{N}$. Thus $y^{\prime}(s)=y^{\prime \prime}(s)$ for every $s \in S$ and $\left|f\left(y^{\prime}\right)-f\left(y^{\prime \prime}\right)\right|_{z} \leqslant \varepsilon$, but it is impossible. 
COROLlaRY 2.3. Let $X \subseteq \mathbb{R}^{T}$ be a compact space, $\varphi: X \rightarrow \mathbb{R}^{S}$ be a continuous mapping and $S_{0} \subseteq S$ with $\left|S_{0}\right| \leqslant \aleph_{0}$. Then there exists a set $T_{0} \subseteq T$ with $\left|T_{0}\right| \leqslant \aleph_{0}$ such that $\left.\varphi\left(x^{\prime}\right)\right|_{S_{0}}=\left.\varphi\left(x^{\prime \prime}\right)\right|_{S_{0}}$ for every $x^{\prime}, x^{\prime \prime} \in X$ with $\left.x^{\prime}\right|_{S_{0}}=\left.x^{\prime \prime}\right|_{T_{0}}$.

Proof: Consider a continuous mapping $f: X \rightarrow \mathbf{R}^{S_{0}}, f(x)=\left.\varphi(x)\right|_{s_{0}}$. Note that $\mathbb{R}^{S_{0}}$ is metrisable. Fix a metric $d$ which generates the topology on $\mathbb{R}^{S_{0}}$. Using Proposition 2.2 for $\varepsilon=0$ we obtain that for every $n \in \mathbb{N}$ there exists a finite set $T_{n} \subseteq T$ such that $d\left(f\left(x^{\prime}\right), f\left(x^{\prime \prime}\right)\right) \leqslant 1 / n$ for any $x^{\prime}, x^{\prime \prime} \in X$ with $x^{\prime}\left|T_{n}=x^{\prime \prime}\right|_{T_{n}}$. The set $T_{0}=\bigcup_{n=1}^{\infty} T_{n}$ is to be found.

Let $X$ be a topological space, $(Y, d)$ be a metric space, $f: X \rightarrow Y$ and $A \subseteq X$ be a nonempty set. The real $\omega_{f}(A)=\sup _{x^{\prime}, x^{\prime \prime} \in A} d\left(f\left(x^{\prime}\right), f\left(x^{\prime \prime}\right)\right)$ is called the oscillation of $f$ on $A$, and the real $\omega_{f}\left(x_{0}\right)=\inf _{U \in \mathcal{U}} \omega_{f}(U)$, where $\mathcal{U}$ is the system of all neighbourhoods of $x_{0} \in X$ in $X$, is called the oscillation of $f$ at $x_{0}$.

The following result illustrates relations between the Namioka property and the dependence of mappings upon some quantity of coordinates.

THEOREM 2.4. Let $X$ be a Baire space, $Y \subseteq \mathbb{R}^{T}$ be a compact space and $f$ : $X \times Y \rightarrow \mathbb{R}$ be a separately continuous function. Then the following conditions are equivalent:

(i) $f$ has the Namioka property;

(ii) for every open in $X$ nonempty set $U$ and every $\varepsilon>0$ there exist an open in $X$ nonempty set $U_{0} \subseteq U$ and a set $S_{0} \subseteq T$ with $\left|S_{0}\right| \leqslant \aleph_{0}$ such that $\left|f\left(x, y^{\prime}\right)-f\left(x, y^{\prime \prime}\right)\right| \leqslant \varepsilon$ for every $x \in U_{0}$ and every $y^{\prime}, y^{\prime \prime} \in Y$ with $\left.y^{\prime}\right|_{S_{0}}$ $=\left.y^{\prime \prime}\right|_{s_{0}}$

(iii) for every open in $X$ nonempty set $U$ and every $\varepsilon>0$ there exist an open in $X$ nonempty set $U_{0} \subseteq U$, a finite set $S_{0} \subseteq T$ and $\delta>0$ such that $\mid f\left(x, y^{\prime}\right)$ $-f\left(x, y^{\prime \prime}\right) \mid \leqslant \varepsilon$ for every $x \in U_{0}$ and every $y^{\prime}, y^{\prime \prime} \in Y$ with $\left|y^{\prime}(s)-y^{\prime \prime}(s)\right|$ $<\delta$, if $s \in S_{0}$.

Proof: (i) $\Rightarrow$ (ii). Let $f$ has the Namioka property and $U$ be an open in $X$ nonempty set. Then there exists an open in $X$ nonempty set $U_{0} \subseteq U$ such that $\mid f\left(x^{\prime}, y\right)$ $-f\left(x^{\prime \prime}, y\right) \mid \leqslant \varepsilon / 2$ for every $x^{\prime}, x^{\prime \prime} \in U_{0}$ and every $y \in Y$. Pick an arbitrary point $x_{0} \in U_{0}$. The continuous function $g: Y \rightarrow \mathbb{R}, g(y)=f\left(x_{0}, y\right)$ depends upon countable quantity of coordinates, that is there exists a set $S_{0} \subseteq T$ with $\left|S_{0}\right| \leqslant \aleph_{0}$ such that $g\left(y^{\prime}\right)=g\left(y^{\prime \prime}\right)$ for every $y^{\prime}, y^{\prime \prime} \in Y$ with $\left.y^{\prime}\right|_{S_{0}}=\left.y^{\prime \prime}\right|_{S_{0}}$. For every $x \in U_{0}$ we have

$$
\begin{aligned}
\left|f\left(x, y^{\prime}\right)-f\left(x, y^{\prime \prime}\right)\right| & \leqslant\left|f\left(x, y^{\prime}\right)-f\left(x_{0}, y^{\prime}\right)\right|+\left|f\left(x_{0}, y^{\prime}\right)-f\left(x_{0}, y^{\prime \prime}\right)\right|+\left|f\left(x_{0}, y^{\prime \prime}\right)-f\left(x, y^{\prime \prime}\right)\right| \\
& \leqslant \varepsilon / 2+\varepsilon / 2=\varepsilon .
\end{aligned}
$$

(ii) $\Rightarrow$ (iii) . Fix an open in $X$ nonempty set $U$ and $\varepsilon>0$. By (ii) there exist an open in $X$ nonempty set $U^{\prime} \subseteq U$ and a set $S^{\prime} \subseteq S$ with $\left|S^{\prime}\right| \leqslant \aleph_{0}$ such that $\mid f\left(x, y^{\prime}\right)$ 
$-f\left(x, y^{\prime \prime}\right) \mid \leqslant \varepsilon / 2$ for every $x \in U^{\prime}$ and every $y^{\prime}, y^{\prime \prime} \in Y$ with $\left.y^{\prime}\right|_{S^{\prime}}=\left.y^{\prime \prime}\right|_{S^{\prime}}$. Let $S^{\prime}=\left\{s_{1}, s_{2}, \ldots, s_{n}, \ldots\right\}$. For every $n \in \mathbb{N}$ denote by $F_{n}$ the set of all points $x \in X$ such that $\left|f\left(x, y^{\prime}\right)-f\left(x, y^{\prime \prime}\right)\right| \leqslant \varepsilon$ for every $y^{\prime}, y^{\prime \prime} \in Y$ with $\left|y^{\prime}\left(s_{k}\right)-y^{\prime \prime}\left(s_{k}\right)\right|<1 / n$ by $k=1,2, \ldots, n$. The continuity of $f$ in the variable $x$ implies that all sets $F_{n}$ are closed. By Proposition 2.2 the continuity of $f$ in the variable $y$ implies $U^{\prime} \subseteq \bigcup_{n=1}^{\infty} F_{n}$. Since $X$ is a Baire space, there exist an integer $n_{0} \in \mathbb{N}$ and an open in $X$ nonempty set $U_{0} \subseteq U^{\prime}$ such that $U_{0} \subseteq \operatorname{int}\left(F_{n_{0}}\right)$. It remains to put $\delta=1 / n_{0}$.

(iii) $\Rightarrow$ (i). Suppose that for an open in $X$ nonempty set $U$ and any real $\varepsilon>0$ there exist an open in $X$ nonempty set $U_{0} \subseteq U$ and a finite set $S_{0} \subseteq T$ and a $\delta>0$ such that $\left|f\left(x, y^{\prime}\right)-f\left(x, y^{\prime \prime}\right)\right| \leqslant \varepsilon$ for every $x \in U_{0}$ and every $y^{\prime}, y^{\prime \prime} \in Y$ with $\left|y^{\prime}(s)-y^{\prime \prime}(s)\right|<\delta$, if $s \in S_{0}$. Then for every $\varepsilon>0$ the open set

$$
G_{\varepsilon}=\left\{x \in X:(\forall y \in Y)\left(\omega_{f}(x, y)<\varepsilon\right)\right\}
$$

is dense in $X$. Put $A=\bigcap_{n=1}^{\infty} G_{1 / n}$. Clearly that $f$ is continuous at each point of $A \times Y$. Thus, $f$ has the Namioka property.

PROPOSITION 2.5. Let $X \subseteq \mathbb{R}^{S}$ be a compact space, $f: X \rightarrow \mathbb{R}$ be a continuous function, $T \subseteq S$ be a set such that $f\left(x_{1}\right)=f\left(x_{2}\right)$ for every $x_{1}, x_{2} \in X$ with $\left.x_{1}\right|_{T}=\left.x_{2}\right|_{T}, \varphi: X \rightarrow \mathbb{R}^{T}, \varphi(x)=\left.x\right|_{T}$ and $Y=\varphi(X)$. Then the function $g: Y \rightarrow \mathbb{R}$, $g\left(\left.x\right|_{T}\right)=f(x)$ is continuous.

Proof: Let $y_{0} \in Y, x_{0} \in X$ be such that $\varphi\left(x_{0}\right)=y_{0}$ and $\varepsilon>0$. The set

$$
F=\left\{x \in X:\left|f(x)-f\left(x_{0}\right)\right| \geqslant \varepsilon\right\}
$$

is closed in $X$, thus $F$ is compact. Therefore the set $\varphi(F)$ is compact subset of $Y$, besides $y_{0} \notin \varphi(F)$. Thus the set $V=Y \backslash \varphi(F)$ is a neighbourhood of $y_{0}$. For every $y \in V$ we have $\left|g(y)-g\left(y_{0}\right)\right|<\varepsilon$. Hence $g$ is continuous at $y_{0}$.

\section{VALDIVIA COMPACTS AND THE NAMIOKA PROPERTY}

In this section we establish a result which we shall use in the proof of a generalisation of Theorem 1.2.

Recall that a compact space $Y$ is called a Valdivia compact if $Y$ is homeomorphic to a compact $Z \subseteq \mathbb{R}^{S}$ such that a set $B=\left\{z \in Z:|\operatorname{supp} z| \leqslant \aleph_{0}\right\}$ is dense in $Z$, where supp $f$ means the support $\{x \in X: f(x) \neq 0\}$ of a function $f: X \rightarrow \mathbb{R}$.

THEOREM 3.1. Let $X$ be a Baire space, $Y \subseteq \mathbb{R}^{T}$ be a Valdivia compact, $\varepsilon>0$ and $f: X \times Y \rightarrow \mathbb{R}$ be a continuous in the firsts variable function such that $\omega_{f^{z}}(y)$ $<\varepsilon$ for every $x \in X$ and every $y \in Y$, where $f^{x}: Y \rightarrow \mathbb{R}, f^{x}(y)=f(x, y)$. Then there exist an open in $X$ nonempty set $U_{0}$ and a set $T_{0} \subseteq T$ with $\left|T_{0}\right| \leqslant \aleph_{0}$ such that $\left|f\left(x, y^{\prime}\right)-f\left(x, y^{\prime \prime}\right)\right| \leqslant 3 \varepsilon$ for every $x \in U_{0}$ and every $y^{\prime}, y^{\prime \prime} \in Y$ with $\left.y^{\prime}\right|_{T_{0}}=\left.y^{\prime \prime}\right|_{T_{0}}$. 
Proof: Note that $Y$ is homeomorphic to a compact $Z \subseteq \mathbb{R}^{S}$ such that the set $B=\left\{z \in Z:|\operatorname{supp} z| \leqslant \aleph_{0}\right\}$ is dense in $Z$. Let $\varphi: Y \rightarrow Z$ be a homeomorphism and $g: X \times Z \rightarrow \mathbb{R}, g(x, z)=f\left(x, \varphi^{-1}(z)\right)$. Clearly, $g$ is continuous in the first variable and $\omega_{g^{x}}(z)<\varepsilon$ for every $x \in X$ and every $z \in Z$, where $g^{x}: Z \rightarrow \mathbb{R}, g^{x}(z)=g(x, z)$.

For each $x \in X$ pick a finite covering $\mathcal{W}_{x}$ of $Z$ by open in $Z$ basic sets such that $\omega_{g^{x}}(W)<\varepsilon$ for every $W \in \mathcal{W}_{x}$. For each $W \in \mathcal{W}_{x}$ choose a finite set $R(W) \subseteq S$ such that for every $z^{\prime}, z^{\prime \prime} \in Z$ the conditions $z^{\prime} \in W$ and $z^{\prime \prime}(s)=z^{\prime}(s)$ for any $s \in R(W)$ imply that $z^{\prime \prime} \in W$. For a finite set $S_{x}=\bigcup_{W \in \mathcal{W}_{x}} R(W)$ we have $\left|g\left(x, z^{\prime}\right)-g\left(x, z^{\prime \prime}\right)\right|<\varepsilon$ for every

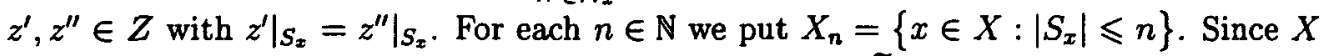
is a Baire space, there exist an open in $X$ nonempty set $\widetilde{U}$ and an integer $n_{0} \in \mathbb{N}$ such that $\widetilde{U} \subseteq \bar{X}_{n_{0}}$.

Show that there exist an open in $X$ nonempty set $U_{0} \subseteq \widetilde{U}$ and a set $S_{0} \subseteq S$ with $\left|S_{0}\right| \leqslant \aleph_{0}$ such that $\left|g\left(x, b^{\prime}\right)-g\left(x, b^{\prime \prime}\right)\right| \leqslant \varepsilon$ for every $x \in U_{0}$ and every $b^{\prime}, b^{\prime \prime} \in B$ with $\left.b^{\prime}\right|_{s_{0}}=\left.b^{\prime \prime}\right|_{s_{0}}$.

Assume that it is false. Pick a set $S_{1} \subseteq S$ with $\left|S_{1}\right| \leqslant \aleph_{0}$ put $U_{1}=\widetilde{U}$. By the assumption, there exist $x_{1} \in U_{1}$ and $b_{1}, c_{1} \in B$ such that $\left|g\left(x_{1}, b_{1}\right)-g\left(x_{1}, c_{1}\right)\right|>\varepsilon$ and $\left.b_{1}\right|_{s_{1}}=\left.c_{1}\right|_{s_{1}}$. Using the continuity of $g$ in the first variable, we find an open in $X$ nonempty set $U_{2} \subseteq U_{1}$ such that $\left|g\left(x, b_{1}\right)-g\left(x, c_{1}\right)\right|>\varepsilon$ for every $x \in U_{2}$. Put $S_{2}=S_{1} \cup\left(\operatorname{supp} b_{1}\right) \cup\left(\operatorname{supp} c_{1}\right)$. By the assumption, there exist $x_{2} \in U_{2}$ and $b_{2}, c_{2} \in B$ such that $\left|g\left(x_{2}, b_{2}\right)-g\left(x_{2}, c_{2}\right)\right|>\varepsilon$ and $b_{2}\left|s_{2}=c_{2}\right|_{s_{2}}$. Doing like that step by step $n_{0}$ times, we obtain a decreasing sequence $\left(U_{n}\right)_{1}^{n_{0}+2}$ of open in $X$ nonempty sets $U_{n}$, an increasing sequence $\left(S_{n}\right)_{n=1}^{n_{0}+2}$ of at most countable sets $S_{n} \subseteq S$ and sequences $\left(b_{n}\right)_{n=1}^{n_{0}+1}$ and $\left(c_{n}\right)_{n=1}^{n_{0}+1}$ of points $b_{n}, c_{n} \in B$ such that the following conditions hold:

(a) $U_{n+1} \subseteq U_{n}$;

(b) $S_{n+1}=S_{n} \cup\left(\operatorname{supp} b_{n}\right) \cup\left(\operatorname{supp} c_{n}\right)$;

(c) $\left.b_{n}\right|_{s_{n}}=c_{n} \mid s_{n}$;

(d) $\left|g\left(x, b_{n}\right)-g\left(x, c_{n}\right)\right|>\varepsilon$ for each $x \in U_{n+1}$

for every $n=1,2, \ldots, n_{0}+1$.

Since $U_{n_{0}+2} \subseteq U_{1}=\tilde{U} \subseteq \bar{X}_{n_{0}}, U_{n_{0}+2} \cap X_{n_{0}} \neq \emptyset$. Pick a point $x_{0} \in U_{n_{0}+2} \cap X_{n_{0}}$ and fix $n \in\left\{1,2, \ldots, n_{0}+1\right\}$. Then $\left|g\left(x_{0}, b_{n}\right)-g\left(x_{0}, c_{n}\right)\right|>\varepsilon$ by Condition (d). The definition of $S_{x_{0}}$ implies $\left.b_{n}\right|_{S_{x_{0}}} \neq\left. c_{n}\right|_{S_{x_{0}}}$. Besides, $\left.b_{n}\right|_{S_{n}}=\left.c_{n}\right|_{S_{n}}$ by Condition (c) and Condition (b) implies that $b_{n}\left|S \backslash S_{n+1}=c_{n}\right|_{S \backslash S_{n+1}} \equiv 0$. Therefore $\left.b_{n}\right|_{S \backslash\left(S_{n+1} \backslash S_{n}\right)}=\left.c_{n}\right|_{S \backslash\left(S_{n+1} \backslash S_{n}\right)}$. Thus $S_{x_{0}} \nsubseteq S \backslash\left(S_{n+1} \backslash S_{n}\right)$, that is $S_{x_{0}} \cap\left(S_{n+1} \backslash S_{n}\right) \neq \emptyset$ for every $n=1,2, \ldots, n_{0}+1$. But this contradicts $\left|S_{x_{0}}\right| \leqslant n_{0}$.

Now we show that $\left|g\left(x, z^{\prime}\right)-g\left(x, z^{\prime \prime}\right)\right| \leqslant 3 \varepsilon$ for every $x \in U_{0} z^{\prime}, z^{\prime \prime} \in Z$ with $\left.z^{\prime}\right|_{S_{0}}=\left.z^{\prime \prime}\right|_{S_{0}}$.

Fix $x \in U_{0}$ and $z^{\prime}, z^{\prime \prime} \in Z$ with $\left.z^{\prime}\right|_{S_{0}}=\left.z^{\prime \prime}\right|_{S_{0}}$. Since the countably compact set $B$ is dense in $Z, \omega_{g^{x}}\left(z^{\prime}\right)<\varepsilon, \omega_{g^{x}}\left(z^{\prime \prime}\right)<\varepsilon$ and $\left|S_{0}\right| \leqslant \aleph_{0}$, there exist $b^{\prime}, b^{\prime \prime} \in B$ such that 
$\left.b^{\prime}\right|_{S_{0}}=\left.z^{\prime}\right|_{S_{0}},\left.b^{\prime \prime}\left|s_{S_{0}}=z^{\prime \prime}\right|\right|_{S_{0}},\left|g\left(x, z^{\prime}\right)-g\left(x, b^{\prime}\right)\right|<\varepsilon$ and $\left|g\left(x, z^{\prime \prime}\right)-g\left(x, b^{\prime \prime}\right)\right|<\varepsilon$. Therefore $\left.b^{\prime}\right|_{S_{0}}=\left.b^{\prime \prime}\right|_{S_{0}}$ and $\left|g\left(x, b^{\prime}\right)-g\left(x, b^{\prime \prime}\right)\right|<\varepsilon$. Thus

$$
\begin{aligned}
\left|g\left(x, z^{\prime}\right)-g\left(x, z^{\prime \prime}\right)\right| & \leqslant\left|g\left(x, z^{\prime}\right)-g\left(x, b^{\prime}\right)\right|+\left|g\left(x, b^{\prime}\right)-g\left(x, b^{\prime \prime}\right)\right|+\left|g\left(x, b^{\prime \prime}\right)-g\left(x, z^{\prime \prime}\right)\right| \\
& <\varepsilon+\varepsilon+\varepsilon=3 \varepsilon .
\end{aligned}
$$

Applying Corollary 2.3 to the compact space $Y$, the mapping $\varphi$ and the set $S_{0}$, we find an at most countable set $T_{0} \subseteq T$ such that $\left.\varphi\left(y^{\prime}\right)\right|_{S_{0}}=\left.\varphi\left(y^{\prime \prime}\right)\right|_{S_{0}}$ for every $y^{\prime}, y^{\prime \prime} \in Y$ with $y^{\prime}\left|r_{0}=y^{\prime \prime}\right|_{r_{0}}$. Then

$$
\left|f\left(x, y^{\prime}\right)-f\left(x, y^{\prime \prime}\right)\right|=\left|g\left(x, \varphi\left(y^{\prime}\right)\right)-g\left(x, \varphi\left(y^{\prime \prime}\right)\right)\right| \leqslant 3 \varepsilon
$$

for every $x \in U_{0}$.

In particular, Theorem 2.4 and Theorem 3.1 imply the following result which was obtained in [3, Corollary 1.2].

COROLlary 3.2. Any separately continuous function on the product of a Baire space and a Valdivia compact has the Namioka property, that is any Valdivia compact is a co-Namioka space.

\section{NAMIOKA SPACES AND $\beta-v$-UNFAVOURABLE SPACES}

In this section we prove a generalisation of Theorem 1.2.

Let $\mathcal{P}$ be a system of subsets of topological space $X$ with the following conditions

$\left(v_{1}\right) \quad \mathcal{P}$ is closed with respect to finite unions;

$\left(v_{2}\right)$ for every set $E \in \mathcal{P}$ and every compact $Y \subseteq C_{p}(X)$ a compact $\varphi(Y)$ is a Valdivia compact, where $\varphi: C_{p}(X) \rightarrow C_{p}(E), \varphi(y)=\left.y\right|_{E}$.

A topological space $X$ is called $\beta-v$-unfavourable if the player $\beta$ has no winning strategy in the $G_{\mathcal{P}}$-game for some system $\mathcal{P}$ with $\left(v_{1}\right)$ and $\left(v_{2}\right)$.

Recall that a compact space $Y$ is called an Eberlein compact if $Y$ is homeomorphic to a compact subset of $C_{p}(X)$ for some compact $X$. A compact space $Y$ is called a Corson compact if $Y$ is homeomorphic to a compact $Z \subseteq \mathbb{R}^{S}$ such that $|\operatorname{supp} z| \leqslant \aleph_{0}$ for every $z \in Z$. It is known that any Eberlein compact is a Corson compact and clearly that any Corson compact is a Valdivia compact.

Proposition 4.1. Let $X$ be a topological space and $\mathcal{K}$ be a system of all nonempty sets $E \subseteq X$ such that for every compact $Y \subseteq C_{p}(X)$ a compact $\varphi(Y)$ is a Corson compact, where $\varphi: C_{p}(X) \rightarrow C_{p}(E), \varphi(y)=\left.y\right|_{E}$. Then $\mathcal{K}$ has $\left(v_{1}\right)$ and $\left(v_{2}\right)$.

Proof: Let $E_{1}, E_{2} \in \mathcal{K}, E=E_{1} \cup E_{2}$, then

$$
\begin{aligned}
\varphi_{1}: C_{p}(X) \rightarrow C_{p}\left(E_{1}\right), & \varphi_{1}(y)=\left.y\right|_{E_{1}}, \\
\varphi_{2}: C_{p}(X) \rightarrow C_{p}\left(E_{2}\right), & \varphi_{2}(y)=\left.y\right|_{E_{2}}, \\
\varphi: C_{p}(X) \rightarrow C_{p}(E), & \varphi(y)=\left.y\right|_{E}
\end{aligned}
$$


and $Y \subseteq C_{p}(X)$ be a compact. Note that a mapping $\psi: \varphi(Y) \rightarrow \varphi_{1}(Y) \times \varphi_{2}(Y)$, $\psi(y)=\left(\left.y\right|_{E_{1}},\left.y\right|_{E_{2}}\right)$, is a homeomorphic embedding. Therefore the compact $\varphi(Y)$ is a Corson compact. Thus $\mathcal{K}$ has $\left(v_{1}\right)$.

The property $\left(v_{2}\right)$ of system $\mathcal{K}$ is obvious.

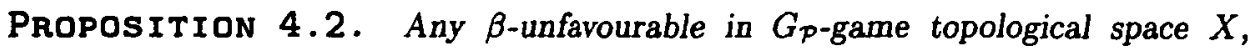
where $\mathcal{P}$ is the system of all bounded subsets of $X$ or $\mathcal{P}$ is a system of all $\mathcal{K}$-countablydetermined subsets of $X$, is a $\beta$ - $v$-unfavourable space.

Proof: Let $E$ be a bounded set in a topological space $X$ and $Y \subseteq C_{p}(X)$ be a compact. Consider a continuous mapping $\psi: X \rightarrow C_{p}(Y)$. Clearly, the set $T=\psi(E)$ is bounded in $C_{p}(Y)$. Therefore by [1, Theorem III.4.1] the closure $\bar{T}$ of $T$ in $C_{p}(Y)$ is a compact. Then a compact $Z=\psi_{1}(Y)$, where $\psi_{1}: Y \rightarrow C_{p}(T), \psi_{1}(y)(t)=t(y)$ for every $y \in Y$ and $t \in T$, is an Eberlein compact, because $Z$ is homeomorphic to a compact subset of $C_{p}(\bar{T})$. Since compacts $Z$ and $\varphi(Y)$, where $\varphi: C_{p}(X) \rightarrow C_{p}(E), \varphi(y)=\left.y\right|_{E}$, are homeomorphic, $\varphi(Y)$ is an Eberlein compact, in particular, $\varphi(Y)$ is a Corson compact.

It follows analogously from [10, Theorem 3.7] that for every $\mathcal{K}$-countably-determined set $E \subseteq X$ and every compact $Y \subseteq C_{p}(X)$ a compact $\varphi(Y)$, where $\varphi: C_{p}(X) \rightarrow C_{p}(E)$, $\varphi(y)=\left.y\right|_{E}$, is a Corson compact.

Thus the systems $\mathcal{P}_{1}$ of all bounded subsets and $\mathcal{P}_{2}$ of all $\mathcal{K}$-countable-determined subsets of the topological space $X$ are contained in the system $\mathcal{K}$, by Proposition 4.1. Therefore any $\beta$-unfavourable space in the $G_{\mathcal{P}_{1}}$-game or in the $G_{\mathcal{P}_{2}}$-game is a $\beta$ unfavourable in $G_{\mathcal{K}}$-game and it is $\beta-v$-unfavourable by Proposition 4.1.

THEOREM 4.3. Any $\beta-v$-unfavourable space is a Namioka space.

Proof: Let $X$ be a $\beta-v$-unfavourable space. Then there exists a system $\mathcal{P}$ of subsets $E$ of topological space $X$ which satisfies $\left(v_{1}\right)$ and $\left(v_{2}\right)$ and such that $X$ is $\beta$ unfavourable in the $G_{\mathcal{p}}$-game.

Assume that $X$ is not a Namioka space. Then there exist a compact space $Y$ and a separately continuous function $f: X \times Y \rightarrow \mathbb{R}$ which does not have the Namioka property. Consider a continuous mapping $\varphi: Y \rightarrow C_{p}(X), \varphi(y)(x)=f(x, y)$. Put $Z=\varphi(Y)$. Clearly, $Z$ is a compact subspace of $\mathbb{R}^{X}$ and a separately continuous mapping $g: X \times Z \rightarrow \mathbb{R}, g(x, z)=z(x)$, does not have the Namioka property. Note that $X$ is a $\beta$-unfavourable space in the Choquet game, that is, $X$ is a Baire space. Therefore by Theorem 2.4 there exist an open in $X$ nonempty set $U_{0}$ and an $\varepsilon>0$ such that for every open in $X$ nonempty set $U \subseteq U_{0}$ and every at most countable set $A \subseteq X$ there exist $x \in U$ and $z^{\prime}, z^{\prime \prime} \in Z$ such that $\left.z^{\prime}\right|_{A}=\left.z^{\prime \prime}\right|_{A}$ and $\left|g\left(x, z^{\prime}\right)-g\left(x, z^{\prime \prime}\right)\right|>\varepsilon$.

Show that for every set $E \in \mathcal{P}$ the set $F(E)=\left\{x \in U_{0}:\left|g\left(x, z^{\prime}\right)-g\left(x, z^{\prime \prime}\right)\right| \leqslant \varepsilon / 8\right.$ for every $z^{\prime}, z^{\prime \prime} \in Z$ with $\left.\left.z^{\prime}\right|_{E}=\left.z^{\prime \prime}\right|_{E}\right\}$ is nowhere dense in $U_{0}$.

Suppose that it is false. Since $U_{0}$ is a Baire space with the topology induced by $X$ and by Proposition 2.1 all sets $F(E)$ are closed in $U_{0}$, there exist a set $E_{0} \in \mathcal{P}$ and 
an open in $X$ nonempty set $V_{0} \subseteq U_{0}$ such that $V_{0} \subseteq F\left(E_{0}\right)$. Suppose $\psi: Z \rightarrow \mathbf{R}^{E_{0}}$, $\psi(z)=\left.z\right|_{E_{0}}$ and $\tilde{Z}=\psi(Z)$. Since the system $\mathcal{P}$ satisfies $\left(v_{2}\right), \widetilde{Z}$ is a Valdivia compact. For every $\tilde{z} \in \tilde{Z}$ choose a point $\tau(\tilde{z}) \in Z$ such that $\psi(\tau(\tilde{z}))=\tilde{z}$. Consider a mapping $h: V_{0} \times \tilde{Z} \rightarrow \mathbb{R}, h(x, \tilde{z})=g(x, \tau(\tilde{z}))$. Since $g$ is continuous in the first variable, $h$ is continuous in the first variable.

Fix $x_{0} \in V_{0}$ and $\tilde{z}_{0} \in \widetilde{Z}$. The set

$$
B=\left\{z \in Z:\left|g\left(x_{0}, z\right)-g\left(x_{0}, \tau\left(\tilde{z}_{0}\right)\right)\right| \geqslant \varepsilon / 4\right\}
$$

is a compact subset of $Z$. Besides, $x_{0} \in F\left(E_{0}\right)$ implies $\widetilde{z}_{0} \notin \psi(B)$. Since $\psi$ is continuous, the set $\psi(B)$ is a compact subset of $\widetilde{Z}$. Thus $\psi(B)$ is a closed subset of $\widetilde{Z}$. Therefore the set $\widetilde{W}=\widetilde{Z} \backslash \psi(B)$ is a neighbourhood of $\tilde{z}_{0}$. Then $\tau(\tilde{z}) \notin B$ for every $\widetilde{z} \in \widetilde{W}$, that is $\left|g\left(x_{0}, \tau(\tilde{z})\right)-g\left(x_{0}, \tau\left(\tilde{z}_{0}\right)\right)\right|=\left|h\left(x_{0}, \widetilde{z}\right)-h\left(x_{0}, \widetilde{z}_{0}\right)\right|<\varepsilon / 4$ for every $\widetilde{z} \in \widetilde{W}$. Hence $\omega_{h x_{0}}\left(z_{0}\right) \leqslant \varepsilon / 4$, where $h^{x_{0}}: \widetilde{Z} \rightarrow \mathbb{R}, h^{x_{0}}(\widetilde{z})=h\left(x_{0}, \widetilde{z}\right)$.

Thus, $h$ satisfies the conditions of Theorem 3.1. Therefore there exist an open in $X$ nonempty set $\widetilde{U} \subseteq V_{0}$ and an at most countable set $A_{0} \subseteq E_{0}$ such that $\mid h\left(x, \tilde{z}^{\prime}\right)$ $-h\left(x, \vec{z}^{\prime \prime}\right) \mid \leqslant 3 \varepsilon / 4$ for every $x \in \widetilde{U}$ and every $\widetilde{z}^{\prime}, \widetilde{z}^{\prime \prime} \in \widetilde{Z}$ with $\left.\widetilde{z}\right|_{A_{0}}=\left.\widetilde{z}^{\prime}\right|_{A_{0}}$.

Pick arbitrary points $x \in \widetilde{U}$ and $z^{\prime}, z^{\prime \prime} \in Z$ such that $\left.z^{\prime}\right|_{A_{0}}=\left.z^{\prime \prime}\right|_{A_{0}}$. Put $\tilde{z}=\psi\left(z^{\prime}\right)$ and $\widetilde{z}^{\prime \prime}=\psi\left(z^{\prime \prime}\right)$. Clearly, $\left.\widetilde{z}^{\prime}\right|_{A_{0}}=\left.\widetilde{z}^{\prime \prime}\right|_{A_{0}}$. Therefore $\left|h\left(x, \widetilde{z}^{\prime}\right)-h\left(x, \widetilde{z}^{\prime \prime}\right)\right| \leqslant 3 \varepsilon / 4$. Since

$$
\left.z^{\prime}\right|_{E_{0}}=\left.\tau(\widetilde{z})\right|_{E_{0}},\left.z^{\prime \prime}\right|_{E_{0}}=\left.\tau\left(\widetilde{z}^{\prime \prime}\right)\right|_{E_{0}}
$$

and

$$
x \in \widetilde{U} \subseteq V_{0} \subseteq F\left(E_{0}\right),\left|g\left(x, z^{\prime}\right)-g(x, \tau(\widetilde{z}))\right|=\left|g\left(x, z^{\prime}\right)-h\left(x, \widetilde{z}^{\prime}\right)\right| \leqslant \varepsilon / 8
$$

and

$$
\left|g\left(x, z^{\prime \prime}\right)-g\left(x, \tau\left(\widetilde{z}^{\prime \prime}\right)\right)\right|=\left|g\left(x, z^{\prime \prime}\right)-h\left(x, \widetilde{z}^{\prime \prime}\right)\right| \leqslant \varepsilon / 8 .
$$

Then

$$
\begin{aligned}
\left|g\left(x, z^{\prime}\right)-g\left(x, z^{\prime \prime}\right)\right| & \leqslant\left|g\left(x, z^{\prime}\right)-h\left(x, \widetilde{z}^{\prime}\right)\right|+\left|h\left(x, \widetilde{z}^{\prime}\right)-h\left(x, \widetilde{z}^{\prime \prime}\right)\right|+\left|h\left(x, \widetilde{z}^{\prime}\right)-g\left(x, z^{\prime \prime}\right)\right| \\
& \leqslant \frac{\varepsilon}{8}+\frac{3 \varepsilon}{4}+\frac{\varepsilon}{8}=\varepsilon .
\end{aligned}
$$

But this contradicts the choice of $U_{0}$.

Thus the set $F(E)$ is nowhere dense in $U_{0}$ for every $E \in \mathcal{P}$.

Describe a strategy for the player $\beta$ in the $G_{\mathcal{P}}$-game. The set $U_{0}$ is the first move of $\beta$. Let $\left(V_{1}, \widetilde{E}_{1}\right)$ be the first move of $\alpha$, where $V_{1} \subseteq U_{0}$ is an open in $X$ nonempty set and $\widetilde{E}_{1} \in \mathcal{P}$. Then $U_{1}=V_{1} \backslash F\left(E_{1}\right)$ is the second move of $\beta$, where $E_{1}=\widetilde{E}_{1}$. If $V_{2} \subseteq U_{1}$ is an open in $X$ nonempty set and $\widetilde{E}_{2} \in \mathcal{P}$ then $U_{2}=V_{2} \backslash F\left(E_{2}\right)$ where $E_{2}=E_{1} \cup \widetilde{E}_{2}$. Continuing the procedure of choice by the obvious manner, we obtain decreasing sequences $\left(U_{n}\right)_{n=0}^{\infty}$ and $\left(V_{n}\right)_{n=1}^{\infty}$ of open in $X$ nonempty sets $U_{n}$ and $V_{n}$ and an 
increasing sequence $\left(E_{n}\right)_{n=1}^{\infty}$ of sets $E_{n} \in \mathcal{P}$ such that $V_{n} \subseteq U_{n-1}, U_{n}=V_{n} \backslash F\left(E_{n}\right)$ and $\widetilde{E}_{n} \subseteq E_{n}$ for every $n \in \mathbb{N}$, where $\widetilde{E}_{n} \in \mathcal{P}$ is the corresponding part of the $n$-th move of $\alpha$. Put $E=\bigcup_{n=1}^{\infty} E_{n}$. Clearly, $\bigcup_{n=1}^{\infty} \widetilde{E}_{n} \subseteq E$. Pick a point $x_{0} \in \bar{E}$. Note that $g\left(x_{0}, z^{\prime}\right)$ $=g\left(x_{0}, z^{\prime \prime}\right)$ for every $z^{\prime}, z^{\prime \prime} \in Z$ with $\left.z^{\prime}\right|_{E}=\left.z^{\prime \prime}\right|_{E}$, that is the continuous function $g^{x_{0}}$ : $Z \rightarrow \mathbb{R}, g^{x_{0}}(z)=g\left(x_{0}, z\right)$, is concentrated on $E$. Using Proposition 2.2, we obtain that there exists a finite set $A \subseteq E$ such that $\left|g\left(x_{0}, z^{\prime}\right)-g\left(x_{0}, z^{\prime \prime}\right)\right|<\varepsilon / 8$ for every $z^{\prime}, z^{\prime \prime} \in Z$ with $\left.z^{\prime}\right|_{A}=\left.z^{\prime \prime}\right|_{A}$. Pick $n_{0} \in \mathbb{N}$ such that $A \subseteq E_{n_{0}}$. Then $x_{0} \in F\left(E_{n_{0}}\right)$ therefore $x_{0} \notin$ $U_{n_{0}}$. Thus $x_{0} \notin \bigcap_{n=0}^{\infty} U_{n}$ and $\bar{E} \cap\left(\bigcap_{n=0}^{\infty} U_{n}\right)=\emptyset$. In particular, $\overline{\left(\bigcup_{n=1}^{\infty} \tilde{E}_{n}\right)} \cap\left(\bigcap_{n=0}^{\infty} U_{n}\right)=\emptyset$. Hence the strategy described above is a winning strategy for $\beta$ in the $G_{\mathcal{p}}$-game, but it is impossible.

Thus, our assumption is false and the theorem is proved.

\section{REFERENCES}

[1] A.V. Arhangel'skii, Topological function spaces (Kluwer Acadademic Publishers, Dordrecht, 1992).

[2] R. Baire, 'Sur les fonctions de variable reelles', Ann. Mat. Pura Appl. 3 (1899), 1-123.

[3] A. Bouziad, 'Notes sur la propriete de Namioka', Trans. Amer. Math. Soc. 344 (1994), 873-883.

[4] J.P.R. Christesen, 'Joint continuity of separately continuous functions', Proc. Amer. Math. Soc. 82 (1981), 455-461.

[5] G. Debs, 'Points de continuite d'une function separement continue', Proc. Amer. Math. Soc. 97 (1986), 167-176.

[6] O.V. Maslyuchenko, Oscillation of separately continuous functions and topological games, (Ph.D. Thesis, in Ukrainian) (Chernivtsi National University, 2002).

[7] I. Namioka, 'Separate continuity and joint continuity', Pacif. J. Math. 51 (1974), 515-531.

[8] V.I. Rybakov, 'Some class of Namioka spaces', Mat. zametki 73 (2003), 263-268.

[9] J. Saint-Raymond, 'Jeux topologiques et espaces de Namioka', Proc. Amer. Math. Soc. 87 (1983), 489-504.

[10] M. Talagrand, 'Espaces de Banach faiblement $\mathcal{K}$-analytiques', Ann. of Math. 110 (1979), 407-438.

[11] M. Talagrand, 'Espaces de Baire et espaces de Namioka', Ann. of. Math. 270 (1985), 159-164.

Chernivtsi National University

Department of Mathematical Analysis

Kotsjubyns'koho 2

Chernivtsi 58012

Ukraine

e-mail: mathan@chnu.cv.ua 